$\xi=-1$

\title{
An Innovative Data-driven Computational Model to Predict High Blood Pressure based on AAA++
}

\author{
Satyanarayana Nimmala ${ }^{1 *}$, Y. Ramadevi² ${ }^{2}$ B. Ashwin Kumar ${ }^{3}$ \\ ${ }^{1}$ Assoc.Prof., CVRCE/CSE, Hyderabad, India \\ ${ }^{2}$ Prof., CBIT/CSE, Hyderabad, India \\ ${ }^{3}$ Asst.Prof., CVRCE/CSE, Hyderabad, India \\ *Corresponding author E-mail:satyauce234@gmail.com
}

\begin{abstract}
Every tissue of human body needs energy and oxygen for its livelihood. In order to supply energy and oxygen, the heart pumps the blood around the body. When heart pushes the blood against the walls of arteries, it creates some pressure inside the arteries, called as blood pressure. If this pressure is more than the certain level we treat it as high blood pressure (HBP). Nowadays HBP is a silent killer of many across the globe. So here we proposed a new data-driven computational model to predict HBP. Blood Pressure (BP) may be elevated because of many changes such as physical and emotional. In the proposed model we have considered AAA++ (age, anger level, anxiety level, obesity (+), blood cholesterol (+)), for experimental analysis. Our model initially calculates the correlation coefficient (CC) between each risk factor and class label attribute. Then based on the impact of each risk factor value and CC, it assigns the corresponding weight to it. Then proposed model uses risk factor value and its weight to predict whether person becomes a victim of HBP or not. We have used real-time data set for experimental analysis. It consists of 1000 records, which are collected from Doctor C, a Medical Diagnostic center, Hyderabad, India.
\end{abstract}

Keywords: Blood Pressure, Blood Cholesterol, Hypertension, Age, Anxiety, Stress Anger, Obesity

\section{Introduction}

BP is represented as Systolic blood pressure (SBP) over Diastolic blood pressure. If SBP exceeds $140 \mathrm{mmHg}$ or DBP exceeds 90 $\mathrm{mmHg}$ on repeated measurements then it is treated as HBP [3]. If HBP is undiagnosed in earlier time, it may cause a serious health problems such as heart stroke, kidney disease, and brain stroke etc.. There are many reasons which may elevate the BP, like unhealthy diet, lack of physical exercise, excess bad cholesterol, obesity, age, anger, anxiety etc [1]. But in this paper, we have taken AAA++, in elevating the BP. Data is collected from a group of 1000 people aged between 20 and 65. High Blood pressure is (HBP) mainly affected by the Cardiac Output (CO) and Total Peripheral Resistance (TPR) [1]. Mathematically, it can be written as equation 1 .

$\mathrm{BP}=\mathrm{CO} * \mathrm{TPR}$

Where CO represents cardiac output and TPR represents total peripheral resistance.

Table 1: Blood Pressure Range

\begin{tabular}{|c|c|c|c|c|}
\hline BP & Low & Normal & Borderline & High \\
\hline Systolic & $<90$ & $90-130$ & $131-140$ & 140 \\
\hline Diastolic & $<60$ & $60-80$ & $81-90$ & $>90$ \\
\hline
\end{tabular}

We collected age, obesity level, and total blood cholesterol levels of 1000 persons from Doctor C, A medical Diagnostic center Hyderabad, India. Although stress, anger, and anxiety may not spike blood pressure for a longer duration of time, but uncontrolled anger and anxiety may affect relationships, career, mental and physical health. Anger and anxiety levels of same persons are measured using the response of an individual for the set of predefined questions. For anger measurement, we set 10 predefined questions and for anxiety measurement, we set 20 predefined questions. The above said people were interviewed and their response is noted on a scale of 0 to 3 . The mean value of the response, for all the questions on anger and anxiety, is used in the prediction process along with age, obesity and total blood cholesterol levels. Table I represents the level of Hypertension [2].

\section{Impact Factors}

Blood pressure of a person is elevated because of emotional, physical and chemical changes. Physical changes are like age, increase in obesity level and total blood cholesterol. Psychological changes like anger, anxiety, stress, depression, and fear. In this paper, we considered AAA++ to predict whether a person is prone HBP or not. The rest of this section discusses how each A in AAA, and ++ elevates the BP. 


\subsection{Impact of AAA}

Age: Many attributes of human body changes as the person ages. When we age the elasticity nature of the arteries also decreases as they become stiff [3]. In such situation, to pump the blood throughout the body through arteries, the heart has to push the blood using more force. This may, in turn, elevate the blood pressure. Anger: Anger may be the result of impatience, frustration, irritation and many others. The way how anger is handled has a significant effect on heart and mind. Frequent explosive anger may lead to serious consequences like elevated blood pressure, and the rise in the heartbeat and pulse rate. When a person gets angry then the fight or flight mode of Sympathetic Nervous System gets activated. As a response, nerves send more blood to muscles and brain, which elevates the blood pressure [4]. Anger Measurement: We used sample questions for anger measurement such are like, waiting for anything annoys me, gets angry for the delay in completion of any assignment, gets angry if things won't go on my path, and I find difficult to forgive people who did wrong to me. We used 10 such questions and for each question, the answer is marked as one of the following option a) no, never b) yes, rarely c) yes, often d) yes, most of the time. In the data preprocessing phase option, option a is considered as 0 , option $b$ is considered as 1 , option $\mathrm{c}$ is considered as 2 and option d is considered as 3. Mean value of all answers is considered for experimental analysis. Anxiety: The active form of stress may be considered as anxiety, and the active form anxiety may be considered as depression. The person, who is a victim of anxiety, thinks again and again about the worst possible outcome of an ambiguous situation, where the possibility of happening best is more. When a person is anxious, fight or flight mode of Sympathetic Nervous System gets activated [5]. As said earlier it elevates the blood pressure. Anxiety Measurement: Our proposed technique measures the level of anxiety by using the responses obtained from the predefined questionnaire. Sample questions used for anxiety measurement are like, the existence of constant fear about something, facing breathing difficulty often, feeling of not having desired things in life, often scared without the clear reason, often aware of the heartbeat without doing physical exercise, and sense of dryness in the mouth. We used 20 such questions and for each question, the answer is marked as one of the following option a) no, never b) yes, rarely c) yes, often d) yes, most of the time. In the data preprocessing phase option, $a$ is considered as 0 , option $b$ is considered as 1 , option c is considered as 2 and option $\mathrm{d}$ is considered as 3 . We considered mean value of all answers for experimental analysis.

\subsection{Impact of Obesity and Cholesterol}

Obesity: Body Mass Index (BMI) is used to measure to find where you fall on the scale of obesity. BMI is a measure of weight proportionate to height. If BMI value is in between 18.5 and 24.9 is treated as normal. If BMI value is greater than 25 and less than are equal to 30 is treated as overweight. If the BMI value is more than 30 then person treated as obese. Obesity is considered as increased fatty tissue in the body [6]. So for the livelihood of increased fatty tissues heart pumps the blood with some additional force to reach newly formed body tissues, which may spike the blood pressure. Cholesterol: Although for the birth and development of body tissues, cholesterol is needed but too much cholesterol in the body is not good for the well being of human body. Lipoproteins (small packages) are transporters of cholesterol in the human body. Lipoproteins are two types, LDL (low-density lipoprotein) cholesterol which is bad cholesterol and not needed for the body [7]. HDL (high-density lipoprotein) cholesterol is called good cholesterol. Indeed, which is most required cholesterol for the functioning many hormones of the human body. HDL carries cholesterol from all parts of the body back to the liver, where cholesterol is filtered and sent out from the body. If LDL is high, this forms fatty substance inside the arteries [8]. This fatty substance reduces the diameter of arteries and raising the blood pressure.

\section{Back ground work}

This section reveals the existing work carried on each parameter in AAA++.Although the research work is done on age and BP, it is Fail to find the exact risk of age in elevating blood pressure[9]. The existing research work also Fail to find the exact risk of obesity in elevating blood pressure [10], it fail to find the exact risk of total blood cholesterol in elevating blood pressure, Fail to find the exact risk of combined effect of age, obesity and total blood cholesterol in elevating blood pressure [11], it is also Fail to find the exact risk of psychological factors such as anger and anxiety in elevating blood pressure. Table 2 represents performance measures of classifier.

Table 2: Performance measures of classifier

\begin{tabular}{|c|c|}
\hline Accuracy, or Recognition rate & $\frac{\mathrm{TP}+\mathrm{TN}}{(\mathrm{P}+\mathrm{N})}$ \\
\hline Error rate, Misclassification rate & $\frac{\mathrm{FP}+\mathrm{FN}}{(\mathrm{P}+\mathrm{N})}$ \\
\hline Sensitivity, True positive rate, TP, Recall & $\frac{\mathrm{TP}}{\mathrm{P}}$ \\
\hline Specificity, True negative rate & $\frac{\mathrm{TN}}{\mathrm{N}}$ \\
\hline Precision & $\frac{\mathrm{TP}}{\mathrm{TP}+\mathrm{FP}}$ \\
\hline $\begin{array}{c}\text { F, F1, F-score, the Harmonic mean of pre- } \\
\text { cision and Recall }\end{array}$ & $\frac{2 \times \text { precision } \times \text { recall }}{\text { precision+recall }}$ \\
\hline
\end{tabular}

Here, We considered record with class label YES as a positive record, and class label NO as a Negative record. Where P is a total number of positive records and $\mathrm{N}$ is a total number of negative records. TP refers to the positive records which are correctly labeled by the classifier. TN is the negative records which correctly labeled by the classifier. FP is the negative records which improperly labeled as positive. FN is the positive records which incorrectly labeled as negative.

\section{Proposed Architecture}

Although Anger and Anxiety may elevate the blood pressure temporarily repeated activation of these two may lead to long-term blood pressure also. Different factors which influence blood pressure of a person directly or indirectly are shown in Fig. 1. Yellow colour represents that impact AAA is more than the impact of ++ (obesity, total blood cholesterol) in elevating the blood pressure. Fig.1 also represents an increase in blood volume, increase in heart rate, and increase in stroke volume also increases blood pressure. These are normally influenced by sympathetic and parasympathetic nervous system of human body.

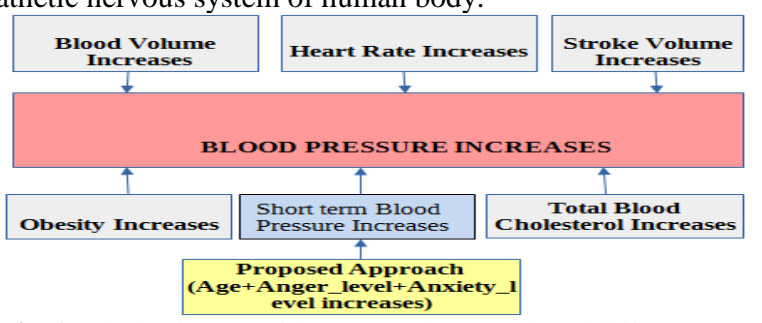

Fig. 1: Block Diagram of the proposed approach and different parameterinfluencing the blood pressures 


\section{Proposed Methodology}

In our proposed methodology initially, we figured out the exact influence of each risk factor in elevating blood pressure. This is done by calculating the Pearson Correlation Coefficient (PCC) between each risk factor and class label attribute. We consider the mean arterial pressure as class label attribute while calculating PCC. Then we used systolic and diastolic pressure to obtain the class label attribute as a binary attribute, which takes either yes or no. if it is yes, means the person is suffering from HBP, if no he or she is not suffering HBP. PCC is calculated using the equation 2. PCC value always lies between -1 and 1 .

$$
P C C=\frac{N \Sigma X Y-\Sigma X \Sigma Y}{\sqrt{\left[N \Sigma X^{2}-(\Sigma X)^{2}\right]\left[N \Sigma Y^{2}-(\Sigma Y)^{2}\right]}}
$$

If PCC value is more means that corresponding risk factor has more influence on high blood pressure so we assign more weight to it. If it has less value means that has less impact on HBP, so we assign less weight to it. Table 3 shows PCC value and the relationship between variables, and Table 4 Shows risk factors and their calculated PCC values.

\subsection{Data set analysis}

We have collected real-time data set consist of 1000 records from Doctor C, a medical diagnostic center Hyderabad, India. Each record consist of age, obesity level, cholesterol level, anger level anxiety level, Systolic blood pressure and diastolic blood pressure.

Table 3: PCC value and Relationship

\begin{tabular}{|c|c|}
\hline PCC value & $\begin{array}{c}\text { The strength of linear relation- } \\
\text { ship between } \mathrm{X} \& \mathrm{Y}\end{array}$ \\
\hline 1 & $\begin{array}{c}\text { Stronger, both moves in the same } \\
\text { direction }\end{array}$ \\
\hline 0 & $\begin{array}{c}\text { Weaker, X, Y relationship is not } \\
\text { linear }\end{array}$ \\
\hline-1 & $\mathrm{X}$ goes up then Y goes down \\
\hline
\end{tabular}

Out of 1000 records, 386 records are of the class label, yes, and 614 records are of class label no. More details of data set are as shown in Table 5. We assigned weight to each risk factor using equation 3.

Table 4: PCC Values between a risk factor and a class label attribute

\begin{tabular}{|c|c|}
\hline Feature or Risk Factor & $\mathrm{PCC}_{\mathrm{i}}$ Value \\
\hline Age $\left(\mathrm{RF}_{1}\right)$ & 0.3976 \\
\hline Obesity level $\left(\mathrm{RF}_{2}\right)$ & 0.3266 \\
\hline Cholesterol level $\left(\mathrm{RF}_{3}\right)$ & 0.2866 \\
\hline Anger level $\left(\mathrm{RF}_{4}\right)$ & 0.5546 \\
\hline Anxiety level $\left(\mathrm{RF}_{5}\right)$ & 0.7014 \\
\hline & Total $=2.2668$ \\
\hline
\end{tabular}

The weight of $\mathrm{RF}_{\mathrm{i}}=\frac{\left(P C C_{i}\right)}{\sum_{i=1}^{5} P C C_{i}} * 100$

Table 6 shows impact factor, its PCC value and weight. The weight of $\mathrm{RF}_{\mathrm{i}}$ (Risk Factor) represents the overall weight of each risk factor in elevating HBP on the scale of 100 units, by considering all possible values of the attribute. But further, these all values of each attribute are divided into a range of values so that we can figure out the exact influence of each attribute based on its value. For example, if age is less than or equal to 30 , its impact is minimal. If age between 30 and 50 its impact is medium and if age greater than 50 its impact is high in elevating the blood pressure. So, selected attribute is further partitioned, based on its value and severity in elevating HBP. So we divided the range of each attribute value as low, medium and high as shown Table 7 . Table 8 shows, how weight is assigned based on the value of impact factor using equation 4,5 , and 6 .

$$
\begin{aligned}
& \text { The Weight of } \mathrm{L}_{\mathrm{i}}=\frac{L_{i} *\left(W e i g h t\left(R F_{i}\right)\right)}{L_{i}+M_{i}+H_{i}} \\
& \text { The weight of } \mathrm{M}_{\mathrm{i}}=\frac{M_{i} *\left(\operatorname{Weight}\left(R F_{i}\right)\right)}{L_{i}+M_{i}+H_{i}} \\
& \text { The weight of } \mathrm{H}_{\mathrm{i}}=\frac{H_{i} *\left(\operatorname{Weight}\left(R F_{i}\right)\right)}{L_{i}+M_{i}+H_{i}}
\end{aligned}
$$

Table 5: Details of attributes

\begin{tabular}{|c|c|c|c|c|c|c|}
\hline Attribute Number & Attribute & Minimum & Maximum & Mean & $\begin{array}{c}\text { Standard Deviation } \\
\text { Type }\end{array}$ \\
\hline 1 & Age & 20 & 65 & 37.898 & 11.021 \\
\hline 2 & Anger level & 0 & 3 & 1.797 & Numeric \\
\hline 3 & Anxiety level & 0 & 3 & 1.141 & 0.618 & 0.664 \\
\hline 4 & Obesity level & 15.5 & 37 & 24.307 & Numeric \\
\hline 5 & $\begin{array}{c}\text { Cholesterol } \\
\text { level }\end{array}$ & 102 & 258 & 168.129 & 31.39 & Numeric \\
\hline
\end{tabular}

\begin{tabular}{|c|c|c|c|c|c|c|}
\hline Attribute & \multicolumn{3}{|c|}{ Range of values } & \multicolumn{3}{|c|}{ Impact based on Range } \\
\hline age & $<=30(\mathrm{L1})$ & $>30 \& \&<=50(\mathrm{M} 1)$ & $>50(\mathrm{H} 1)$ & $\mathrm{L} 1=1$ & $\mathrm{M} 1=2$ & $\mathrm{H} 1=3$ \\
\hline Obesity level & $<=25(\mathrm{~L} 2)$ & $>25 \& \&<=30(\mathrm{M} 2)$ & $>30(\mathrm{H} 2)$ & $\mathrm{L} 2=1$ & $\mathrm{M} 2=2$ & $\mathrm{H} 2=3$ \\
\hline Cholesterol level & $<=150(\mathrm{~L} 3)$ & $>150 \& \&<=200(\mathrm{M} 3)$ & $>200(\mathrm{H} 3)$ & $\mathrm{L} 3=1$ & $\mathrm{M} 3=2$ & $\mathrm{H} 3=3$ \\
\hline Anger level & $<=1(\mathrm{~L} 4)$ & $>1 \& \&<2(\mathrm{M} 4)$ & $>2(\mathrm{H} 4)$ & $\mathrm{L} 4=1$ & $\mathrm{M} 4=2$ & $\mathrm{H} 4=3$ \\
\hline Anxiety level & $<=1($ L5) & $>1 \& \&<=2$ (M5) & $>2(\mathrm{H} 5)$ & $\mathrm{L} 5=1$ & M5=2 & $\mathrm{H} 5=3$ \\
\hline
\end{tabular}

Table 6: PCC value and its associated weight

\begin{tabular}{|c|c|c|}
\hline \multicolumn{1}{|c|}{ Table 6: PCC value and its associated weight } \\
\hline Attribute & PCC Value & Weight RF \\
\hline Age & 0.3976 & 17.54 \\
\hline Obesity level & 0.3266 & 14.40 \\
\hline Cholesterol level & 0.2866 & 12.64 \\
\hline Anger level & 0.5546 & 24.44 \\
\hline Anxiety level & 0.7014 & 30.94 \\
\hline
\end{tabular}

Table 7: Impact based on the value of the attribute 
Table 8: Weight of attribute based on the value

\begin{tabular}{|c|c|c|c|c|}
\hline Attribute & \multicolumn{3}{|c|}{ Weight distribution to risk factors based on the attribute value } & \multirow{2}{*}{$\begin{array}{c}\text { Total Weigh } \\
17.54\end{array}$} \\
\hline Age & $\begin{array}{c}<=30 \\
\text { Then } \\
\mathrm{WL}_{1}=2.9233\end{array}$ & $\begin{array}{c}>30 \& \&<=50 \\
\text { Then } \\
\mathrm{WM}_{1}=5.8466\end{array}$ & $\begin{array}{c}>50 \\
\text { Then } \\
\mathrm{WH}_{1}=8.77\end{array}$ & \\
\hline Obesity Level & $\begin{array}{c}<=25 \\
\text { Then } \\
\mathrm{WL}_{2}=2.4\end{array}$ & $\begin{array}{c}>25 \& \&<=30 \\
\text { Then } \\
\mathrm{WM}_{2}=4.8\end{array}$ & $\begin{array}{c}>30 \\
\text { Then } \\
\mathrm{WH}_{2}=7.2\end{array}$ & 14.40 \\
\hline Cholesterol Level & $\begin{array}{c}<=150 \\
\text { Then } \\
\mathrm{WL}_{3}=2.1066\end{array}$ & $\begin{array}{c}>150 \& \&<=200 \\
\text { Then } \\
\mathrm{WM}_{3}=4.2133\end{array}$ & $\begin{array}{c}>200 \\
\text { Then } \\
\mathrm{WH}_{2}=6.32\end{array}$ & 12.64 \\
\hline Anger Level & $\begin{array}{c}<=1 \\
\text { Then } \\
\mathrm{WL}_{4}=4.073\end{array}$ & $\begin{array}{c}>1 \& \&<2 \\
\text { Then } \\
\mathrm{WM}_{4}=8.1466\end{array}$ & $\begin{array}{c}>2 \\
\text { Then } \\
\mathrm{WH}_{4}=12.22\end{array}$ & 24.44 \\
\hline Anxiety Level & $\begin{array}{c}<=1 \\
\text { Then } \\
\mathrm{WL}_{5}=5.1566\end{array}$ & $\begin{array}{c}>1 \& \&<=2 \\
\text { Then } \\
\mathrm{WM}_{5}=10.3133\end{array}$ & $\begin{array}{c}>2 \\
\text { Then } \\
\mathrm{WH}_{5}=15.47\end{array}$ & 30.94 \\
\hline
\end{tabular}

Table 9: Thresh hold set up based on performance measures

\begin{tabular}{|c|c|c|c|c|c|c|}
\hline $\begin{array}{c}\text { Thresh hold } \\
\text { value(Y) }\end{array}$ & Accuracy & Tp rate & Fp rate & Precision & Recall \\
\hline 28 & 61.6 & 0.997 & 0.955 & 0.610 & 0.997 \\
\hline 30 & 62.4 & 0.493 & 0.180 & 0.804 & 0.493 \\
\hline 32 & 63 & 0.673 & 0.435 & 0.699 & 0.673 & 0.612 \\
\hline 35 & 66.6 & 0.867 & 0.635 & 0.672 & 0.687 & 0.900 \\
\hline 40 & 67.2 & 0.900 & 0.670 & 0.668 & 0.757 \\
\hline 45 & 69.6 & 0.873 & 0.570 & 0.697 & 0.767 \\
\hline 47 & 73 & 0.840 & 0.435 & 0.743 & 0.775 \\
\hline 49 & 78 & 0.847 & 0.320 & 0.799 & 0.789 \\
\hline $\mathbf{5 3}$ & $\mathbf{8 0 . 8}$ & $\mathbf{0 . 8 7 0}$ & $\mathbf{0 . 2 8 5}$ & $\mathbf{0 . 8 2 1}$ & 0.847 \\
\hline 58 & 79.6 & 0.877 & 0.325 & 0.802 & $\mathbf{0 . 8 7 0}$ & 0.877 \\
\hline
\end{tabular}

\subsection{Data normalization}

In our Data set anger, anxiety levels are measured on the scale of $[0,3]$, but age, obesity, and cholesterol levels are measured on different scales. So to bring them on to the scale of $[0,3]$, so that weight of each factor has equal impact on class label attribute, we used normalization technique. There are different normalization techniques but we have used min_max normalization in normalizing age, obesity and cholesterol levels.

$\mathrm{X}_{\text {new }}=\frac{o X-\min (\mathrm{x})}{\max (x)-\min (x)} *($ new_max - new_min $)+$ new_min

Here $\mathrm{X}_{\text {new }}$ is the new value calculated for the observed value $(\mathrm{oX})$, $\max (\mathrm{x})$ is the maximum value of the observed attribute, $\min (\mathrm{x})$ is the minimum value of the observed attribute, new_max represents the maximum value of new range and new_min represents minimum value of the new range. Using the above equation all the values of age, obesity, and cholesterol levels are normalized in the new range of $[0,3]$, such that all the five attributes are taking values between $[0,3]$.

\subsection{Thresh hold set up Using prediction function}

We divided the data set into two sets, one is training data set and another is testing data set. Training data set has been used to set the thresh hold value for prediction. The following Table shows the Accuracy, True positive rate, True negative rate, precision, recall and F-measure values for different thresh hold values of $\mathrm{Y}$ using equation 8 .

$\mathrm{Y}=\sum_{i=1}^{n} w_{i} * x_{i}$
Where $\mathrm{w}_{\mathrm{i}}$ is the weight of the attribute and $\mathrm{x}_{\mathrm{i}}$ is a normalized value of the attribute. As we considered 5 attributes for HBP prediction, so here $n=5$. Using Table 9, we set thresh hold limit as 53. If the calculated value of $\mathrm{Y}$ using equation 8 exceeds 53 then, that record is predicted as yes otherwise no. Table 9 shows different thresh hold values and accuracy for each. We obtained maximum accuracy when thresh hold value is 53. So we have taken 53 as thresh hold value for $\mathrm{Y}$ in predicting test data set, and we got $81 \%$ accuracy.

\section{Experimental Results and Analysis}

For the experimental analysis, we have collected real-time data set from 1000 people. Each person data is considered as one record, for each record age of a person, anger level, anxiety level, obesity, blood cholesterol, systolic and diastolic blood pressures are recorded. Based on the systolic and diastolic blood pressure values we calculated class label attribute. Mean value of answers obtained from the questionnaire used for measuring anger, anxiety levels are taken for experimental analysis. Table 1 is used to convert systolic and diastolic blood pressure values to get the class label attribute. Performance of classifier, the details of the dataset are as shown in Table 2, and Table 5. Data collection is done manually by interacting with the people using the questionnaire as mentioned. We have used a data mining tool WEKA (Waikato Environment for Knowledge Analysis) for experimental analysis in data preprocessing phase. It is open source software; consist of many machine learning and data mining algorithms. WEKA processes the input data using ARFF (Attribute file format). So data collected is converted into an arff file in the data preprocessing phase. Fig. 2, 3, 4 red x represents data record predicted as yes and black $\mathrm{x}$ represents data record predicted as no. In these figures, the prediction is done by selecting two attributes using our proposed approach. 


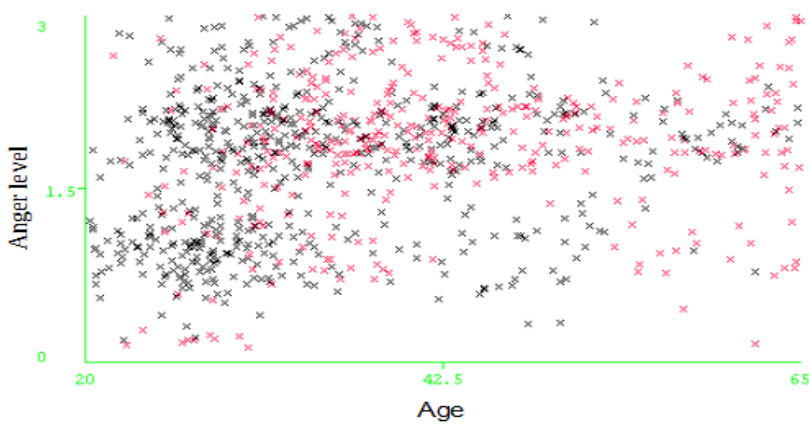

Fig. 2: HBP prediction using Age and Anger

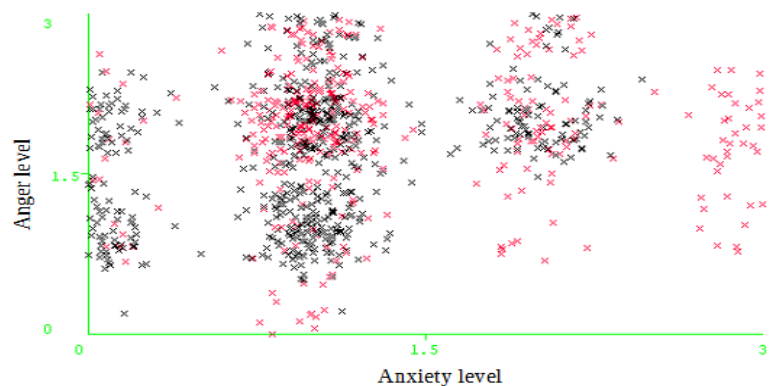

Fig. 4: HBP prediction using Anxiety and Anger

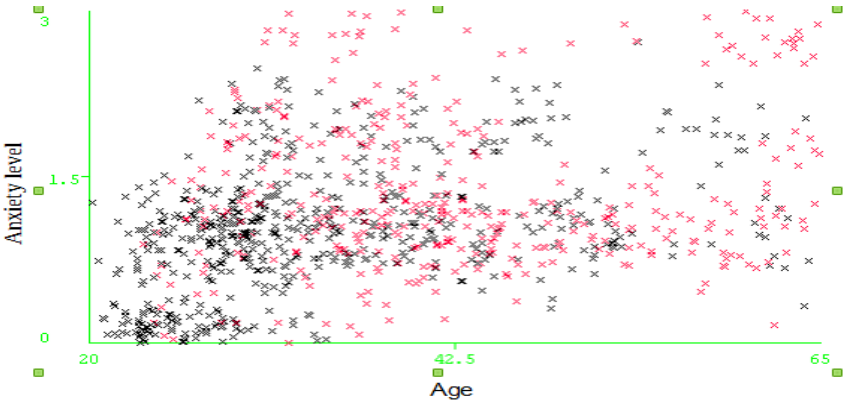

Fig. 3: HBP prediction using Age and Anxiety

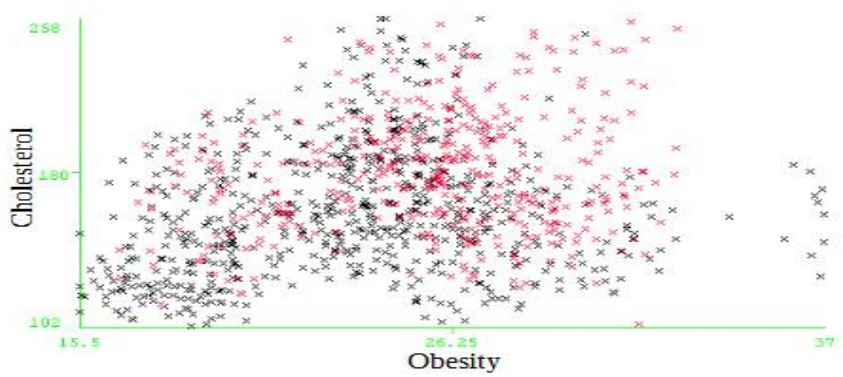

Fig. 5:. HBP prediction using Obesity and cholesterol

Table 10: Class-wise Accuracy Details Using the Proposed approach

\begin{tabular}{|c|c|c|c|c|c|}
\hline Class & TP Rate & FP Rate & Precision & F-Measure \\
\hline YES & 0.715 & 0.130 & 0.786 & 0.715 \\
\hline NO & 0.808 & 0.223 & 0.807 & 0.749 \\
\hline
\end{tabular}

\section{Conclusion}

In our paper, we considered obesity, cholesterol levels, age, anger and anxiety levels of a person for experimental analysis. Experiments revealed that the anger and anxiety levels are playing a vital role in elevating the blood pressure of a person. Experiments also unfold, if person age is more than 40 and obesity level is more than 30 he is more prone to HBP. However if cholesterol level is more than 200 and if is worried and anxious he is also a victim of HBP. We observed $\mathrm{N}$ number of interesting patterns from the experimental analysis but all are not listed here. In future; we would like to consider other attributes such as gender, smoking, job satisfaction, marital status, and alcohol consumption to improve the prediction performance of the proposed classifiers.

\section{Acknowledgments}

We thank Doctor C, a Medical Diagnosis Center, Hyderabad for their support in providing Data Set. We also thank Dr. Seetharam, Cardiologist, Yashoda hospitals, Hyderabad on improving this manuscript.

\section{References}

[1] Satyanarayana, N., Ramalinga swamy, CH., Ramadevi, Y High blood pressure prediction based on AAA using J48 classifier. In 2018 IEEE conference on signal processing and communication Engineering(SPACES) 2018.

[2] Mikko Peltokangas., Antti Vehkaoja., J armo Verho. 2017. Age Dependence of Arterial Pulse Wave Parameters Extracted from Dynamic blood pressure and Blood Volume Pulse Waves. IEEE journal of biomedical and Health informatics, 21: 142-149.

[3] Satyanarayana, N., Ramalinga swamy, CH., Ramadevi, Y. 2014. Survey of Classification Techniques in Data mining, International Journal Innovative Science, Engineering
\&Technology, 1:268-278.

[4] A. Alwan. 2011 Global status report on noncommunicable diseases 2010, World Health Organization, 2011.

[5] Julie K.K. Vishram., Anders Borglykke., Anne H. Andreasen., Jorgen Jeppesen., Hans Ibsen. 2012. Impact of Age on the Importance of Systolic and Diastolic Blood Pressures for Stroke Risk, Hypertension, 60:1117- 1123.

[6] Raghupathy Anchala., Nanda K. Kannuri ., Hira Pant . 2014. Hypertension in India: a systematic review and meta- analysis of prevalence, awareness, and control of hypertension, Journal of Hypertension, 32:1170-1177.

[7] World Health Organization [available online at]: http://www.who.int/gho/ncd/risk_factors/blood_pressure prevaletext/en/.

[8] Ilse L. Mertens., Luc F. Van Gaal. 2000. Overweight, Obesity, and Blood Pressure The Effects of Modest Weight Reduction. Obesity research, 8:270-278.

[9] Richard N. Re. 2009. Obesity Related Hypertension. The Ochsner Journal, 9:133-136.

[10] Mohamad Forouzanfar., Hilmi R. Dajani., Mohamad Forouzanfar., hilmi R. Dajani., Voicu Z. Groza., Miodrag Bolic.,Sreeraman Rajan. ,and Izmail Batkin.2015. Oscillometric Blood pressure Estimation: Past, Present, and Future. IEEE reviews in biomedical Engineering, 8:44-61.

[11] National Heart, lung and blood institute [available online at] https://www.nhlbi.nih.gov/health/healthtopics/hbc/Causes.

[12] Meenakshi Kalyan., Shubhangi A. Kanitkar. 2015. Ultrasonogra-phic assessment of abdominal fat and its correlation with blood pressure. International Journal of Biomedical and Advance Research, 6:259-263. 